\title{
Labyrinthe
}

$33 \mid 2009$ (2)

"Patates chaudes " : poétique, savoirs, politique

\section{Les ratés de la famille}

Balzac et les formes sociales de la sexualité de Michael Lucey

\section{Marion Mas}

\section{(2) OpenEdition}

\section{Journals}

Édition électronique

URL : http://journals.openedition.org/labyrinthe/4034

DOI : 10.4000 /labyrinthe.4034

ISSN : 1950-6031

Éditeur

Hermann

\section{Édition imprimée}

Date de publication : 23 octobre 2009

Pagination : 141-146

ISBN : 978-2-7056-6976-8

Référence électronique

Marion Mas, «Les ratés de la famille », Labyrinthe [En ligne], 33 | 2009 (2), mis en ligne le 23 octobre 2011, consulté le 21 septembre 2020. URL : http://journals.openedition.org/labyrinthe/4034 ; DOI : https://doi.org/10.4000/labyrinthe.4034 


\section{LES RATÉS DE LA FAMILLE : \\ BALZAC ET LES FORMES SOCIALES DE LA SEXUALITÉ DE MICHAEL LUCEY1}

Marion Mas

Dans Les ratés de la famille, Michael Lucey part du constat que les romans de Balzac représentent un monde où il n'existe pas de forme fixe de la famille, mais diverses modalités d'intrication entre la sexualité et les régimes juridique et institutionnel de l'héritage. Le roman balzacien est lu comme un espace de confrontation entre, d'une part, différents discours sur la famille et entre, d'autre part, ces discours et les comportements, normés ou "déviants ", des personnages. Lucey remarque également que les intrigues et les interrelations entre les personnages permettent de figurer la manière dont ces discours et ces comportements sont perçus. Cet ensemble à triple détente produit alors un troisième niveau de discours, théorique, qui éclaire, d'un côté, les intérêts économiques, symboliques et psychologiques promus par l'ensemble des discours sur la famille et, d'un autre côté, les catégories qui gouvernent la perception de comportements a-normés, et leur insuffisance à en rendre compte. En prenant la « curiosité sociologique et épistémologique » de Balzac pour objet de son étude, Lucey interroge donc l'institution famille, en train de se recomposer, et la construction des identités sexuelles (le célibat, l'homosexualité, des identités non nommables) dans leurs rapports réciproques.

La première partie de l'ouvrage s'intéresse aux relations familiales atypiques tandis que la seconde se concentre sur les relations entre individus de même sexe. Lucey aborde l'œuvre balzacienne par le prisme de la sexualité, dont il fait un motif herméneutique et un instrument d'analyse littéraire, en mobilisant des références théoriques antagonistes: Bourdieu et le Foucault de La Volonté de savoir, que les gender studies lui permettent peu ou prou d'articuler.

En effet, les gender studies retiennent de la sociologie que la sexualité, loin d'être une catégorie naturelle, est entièrement construite par divers paramètres inextricablement imbriqués les uns dans les autres (les

1. Paris, Fayard 2008, traduit de l'anglais (2003) par Didier Éribon 
« comportements émotionnels, [le] rapport à la reproduction biologique, [le] rapport à la production économique [et] à la reproduction des formes sociales telles que la famille ${ }^{1} »$, note 9, p. 304). Mais elles postulent également que ce n'est que par le langage, par la littérature en particulier, que la sexualité est investie de sens. Autrement dit, c'est le discours qui ordonne les rapports individuels à partir des catégories de masculin et de féminin. Or on sait que dans La Volonté de savoir, Foucault montre que le discours est le lieu où se forgent et où se transforment, par leur action réciproque, les relations de « savoir-pouvoir » à propos de la sexualité 2 .

Dans la première partie de son livre, Lucey étudie les trajectoires des « personnages queer. » Il précise que cette caractérisation « n'implique rien de particulier quant à la sexualité des personnes concernées. [Elle] dénote plutôt une situation sociale non normative ${ }^{3} »($ note 9, p. 304) au regard des pratiques de la famille et de l'identité sexuelle. Le destin malheureux de ces personnages (à la sexualité indéterminée ou bien célibataires), qui refusent d'intégrer les dispositifs d'alliance, permet de mettre en lumière, a contrario, la norme familiale, bourgeoise et hétérosexuelle, en train de s'imposer. Faisant implicitement référence au dispositif de sexualité décrit par Foucault, Lucey montre que ce nouveau modèle est régi par l'articulation des transformations du régime successoral imposées par le Code civil (dont il fait une lecture précise) et de ce qu'il appelle « l'idéologie reproductrice. » Pour autant, le critique ne s'intéresse aux effets $\mathrm{du}$ « branchement » du dispositif d'alliance et du dispositif de sexualité que sur l'institution familiale, contrairement à Foucault, qui envisage la perspective globale d'une politique administrative de la gestion des corps. Il appuie sa démonstration sur les études monographiques d'Ursule Mirouët, d'Eugénie Grandet, du Père Goriot, de Pierrette et du Cousin Pons. Pour ces analyses de détail, il emprunte les notions d'habitus, d'« hexis corporelle » et de champ à Bourdieu, afin de mettre en évidence la variété des positions à l'égard de la norme que le roman balzacien offre

\section{Ibidem.}

2. Pour Foucault, la sexualité n'est pas une donnée de la nature que le pouvoir essaierait de mater ou que le savoir essaierait de dévoiler. La sexualité au contraire est « le nom qu'on peut donner à un dispositif historique [...] où la stimulation des corps [...], l'incitation des discours, la formation des connaissances, le renforcement des contrôles et des résistances, s'enchaînent les uns avec les autres, selon quelques grandes stratégies de savoir et de pouvoir. », Michel Foucault, Histoire de la sexualité I, La Volonté de Savoir, Paris, Gallimard, 1976, p. 139.

3. Michael Lucey, Op. cit. 
à ses personnages. D'un côté, cette série d'études montre, certes, que les structures familiales parallèles inventées par les personnages, pour faire échapper des relations économiques et affectives aux contraintes du Code, sont toujours exclues du système d'héritage dès lors qu'elles refusent le schéma d'alliance hétérosexuelle. Celui-ci, intimement lié à des intérêts économiques, fonde seul la norme familiale. Mais d'un autre côté, le motif de la sexualité atteste que l'identité sexuelle des personnages balzaciens, loin d'être fixée à l'avance et de pouvoir constituer, à cet égard, le socle des familles et de la société, se fabrique au contraire dans le rapport entre des forces extérieures, les pratiques et les « dispositions » des individus. En effet, le corps « imprime » un certain nombre de comportements et de normes sociales. Dans cette perspective, la sexualité est pensée comme une forme de connaissance pratique et immédiate du monde, soustraite à « l'acte intentionnel de déchiffrement conscient », selon les termes de Pierre Bourdieu cités par Lucey (p. 29). Ainsi, les intrigues révèlent que les dispositions corporelles acquises par les personnages peuvent aussi bien jouer contre leurs intérêts propres qu'augmenter leur lucidité et leur permettre de manœuvrer socialement: les institutions n'ont pas le même effet normatif suivant la position qu'occupent les personnages dans le champ social.

Enfin, l'effort du roman balzacien pour saisir la complexité et la multiplicité de ces relations en transformation montre qu'il existe d'autres modèles disponibles que le seul modèle bourgeois pour penser la famille dans son rapport à la sexualité.

Dans un « interlude », Lucey aborde (entre autres) la question de l'homosexualité supposée de Balzac, dans une perspective bourdieusienne, pour montrer que les relations entre personnages du même sexe sont intégrées dans des enjeux de positionnement social et de classe. Cette grille de lecture guide par exemple l'analyse de La Fille aux yeux d'or, dont le romanesque est interprété comme la formulation réciproque d'une identité homosexuelle et d'un ethos aristocratique.

La seconde partie de l'ouvrage s'ouvre sur un hommage explicite à Foucault, auquel Lucey emprunte sa théorie du pouvoir, pour proposer une hypothèse méthodologique originale. Il fait jouer en tension l'antagonisme des références convoquées (Bourdieu et Foucault) pour analyser les « amitiés déviantes » des personnages (Pons, la cousine Bette et Vautrin) comme des «formes sociales » de la sexualité. Autrement dit, 
il montre que la sexualité peut se loger là où on ne l'attend pas, dans d'autres formes de relations sociales instituées (comme l'amitié ou la relation paternelle par exemple). Grâce à la notion de « forme sociale ", Lucey dépasse Bourdieu et les propositions de l'interlude, en montrant que la sexualité des personnages et la manière de figurer cette sexualité n'est pas simplement une prise de position visant un « écart différentiel »; elle n'est pas uniquement une manière de se soustraire à l'idéologie bourgeoise par la mobilisation d'un « capital déviant». Par ailleurs, la catégorie plastique de « forme sociale » permet à Lucey de s'approprier l'idée de transformation, centrale dans la théorie foucaldienne du pouvoir, mais pour s'écarter à nouveau de Foucault. En effet, si la forme sociale dans laquelle se loge la sexualité est une « matrice de transformations » des relations de pouvoir, ce n'est pas en tant qu'elle produit de nouveaux discours de savoir sur la sexualité (et, partant, de nouvelles relations de « savoir-pouvoir »), mais en tant qu'elle neutralise les relations de « savoir-pouvoir». Le détournement des formes sociales instituées au profit d'autres pratiques relationnelles empêche précisément tout discours de savoir à l'égard de la sexualité. La forme sociale dans laquelle se loge une sexualité déviante devient donc un défi à la production de relations de « savoir-pouvoir » et peut s'analyser comme un foyer de résistance (impliqué par les rapports mêmes de pouvoir à l'œuvre dans le dispositif de sexualité) à l'homogénéisation en train de se mettre en place.

Lucey analyse la manière dont le roman formule les « amitiés déviantes » des personnages pour montrer, en dernière instance, que c'est dans le discours du roman que se transforment les relations de pouvoir: la littérature, en refusant de catégoriser, capte de l'inassimilable pour neutraliser les rapports de pouvoir autour de la sexualité. Ce faisant, le roman balzacien produit un discours sur la sexualité qui n'est pas un discours de pouvoir.

D'où les interprétations contradictoires et passionnées suscitées par le personnage de Vautrin par exemple. S'intéressant à la question de son homosexualité, Lucey déplace les enjeux de pouvoir liés à la sexualité au niveau de la réception critique du personnage. Selon lui, la variation historique des lectures critiques renvoie non seulement à l'inscription d'un « agent social » particulier dans un champ social particulier, et donc, à des attitudes diverses, mais également à des stratégies de l'agent en question « pour établir sa trajectoire dans ce champ » (p. 289). 
Enfin, pour conforter l'idée selon laquelle la littérature est une force disruptive, Lucey fait dialoguer Balzac et Proust. L'attention prêtée par ces deux auteurs à la variété, au dynamisme et à la contingence de formes sexuelles « parias » s'oppose à toute tentative de discours simplificateur et homogénéisant à leur propos et impose une lecture historique des « formes sociales » (du mariage, de l'amitié, des institutions).

La méthode de Lucey sert aussi une lecture critique de la psychanalyse. Tout d'abord, elle éclaire l'approche freudienne de la sexualité, en montrant que par sa normativité, celle-ci relève finalement du même « moment historique » que celui que les récits de Balzac épinglent. Ensuite, elle pointe l'insuffisance des approches psychanalytiques de l'œuvre de Balzac, dominantes dans la critique américaine contemporaine, qui escamotent nécessairement le travail d'analyse historique des formes familiales et sexuelles auquel se livre le romancier. Par ailleurs, Lucey s'inscrit dans une tendance actuelle de la critique balzacienne, qui consiste à lire Balzac comme un «pré-sociologue » et que l'on peut considérer comme une manière renouvelée d'aborder le courant esthétique réaliste. Enfin, Balzac et les ratés de la famille apporte un éclairage nouveau sur les rapports entre Code civil et fiction: en appliquant la lecture du Code au thème de la sexualité plutôt qu'à celui du mariage, Lucey souligne l'importance décisive de la catégorie d'héritier pour le roman balzacien.

Toutefois, bien que l'ouvrage appuie son propos sur des analyses littéraires pertinentes (on pense par exemple à celle, brillante, de la circulation du châle de la cousine Bette comme motif narratif signalant l'impossibilité d'échapper au modèle matrimonial et patriarcal), on regrette que les romans soient trop souvent considérés comme autant de documents sociologiques. Il manque une analyse poétique d'ensemble, de la manière, par exemple, dont Balzac s'approprie le genre romanesque comme moyen privilégié de mettre en texte le social.

Mais le livre de Lucey demeure une lecture très stimulante. Il insiste sur le refus du texte à intégrer les catégories toutes faites. Le roman en effet, met en abyme l'illisibilité d'un certain nombre de relations « sans nom » entre personnes de même sexe, les rendant par-là inassimilables, non seulement par les différents discours sociaux (médicaux, familiaux, juridiques), mais également par les différentes approches disciplinaires: la sociologie et les gender studies en l'occurrence. Dans le même temps cependant, le pari de la lecture « interdisciplinaire » permet de donner 


\section{Labyrinthe, $n^{\circ} 33$}

sens à cet « inassimilable » représenté par le roman, en lui faisant poser des « questions radicales » et notamment celle-ci : que serait la sexualité si elle n'était plus liée à l'héritage et à la famille? 